\title{
Perfil de los pacientes rehabilitados por medio de prótesis atendidos en servicio público de salud en Ribeirão Preto, Brasil
}

\section{Patients profile rehabilitated with dental prosthesis assisted by the health public service in Ribeirão Preto, Brazil}

\author{
de Andrade Lima Chaves C*, Alves Gomes E*, Faria T*, Rocha Correa da Silva S*, \\ de Souza Filho CB*, Borie E*****, Corrêa Silva Sousa YT*
}

\begin{abstract}
RESUMEN
La prevalencia de sexo y el padrón de edad relacionado a los diferentes tipos de rehabilitaciones protésicas son variables importantes que deben ser estudiadas. De esta manera, el objetivo de este estudio fue evaluar el perfil de edad y género en pacientes rehabilitados con prótesis dentales en un curso de Odontología durante un periodo de cinco años. Fueron evaluados 638 fichas de pacientes que recibieron prótesis parcial fija (PPF), prótesis parcial removible (PPR) y prótesis total (PT). Las prótesis de los pacientes rehabilitados con PPR fueron clasificadas en dentomucosoportada (DM) o dentosoportadas (D). Para el análisis de los resultados, los pacientes fueron divididos en 2 grupos: A) $\geq 50$ años y B) $<50$ años. Los datos fueron analizados estadísticamente por el test exacto de Fisher o chi-cuadrado $(p \leq 0,05)$. No se observaron diferencias significativas en relación a la edad y tipo de prótesis. Para la PPF $74 \%$ fueron $<50$ años, para la PPR $52 \%, \geq 50$ años y para la PT 92\%, $\geq 50$ años. En todas las prótesis, existió una mayor prevalencia del género femenino. Con relación a la clasificación de las PPRs, 47\% fueron D y 53\% DM, sin identificar diferencias estadísticamente significativas. De esta manera se puede concluir que la necesidad de rehabilitación de un mayor numero de elementos dentales aumenta con la edad, siendo prevalente en todos los periodos de la vida adulta, principalmente, en mujeres.
\end{abstract}

Palabras clave: Prótesis dental, prevalencia, epidemiología.

\section{SUMMARY}

The prevalence of gender and age related pattern of the different types of prosthetic restorations are important variables to be estimated. The aim of this study was to evaluate the profile of age and gender in patients rehabilitated with dental prostheses on School of Dentistry during five years. Were evaluated 638 records of patients who received fixed prosthesis (FP), removable partial (RP) or total dentures (T). Rehabilitated patients with RP had their prostheses classified into dental-mucous-supported (DM) or tooth-supported (D). To analyze the results, patients were divided into 2 groups: A) $\geq 50$ years or B) $<50$ years. Data were statistically analyzed by Fisher's exact test or chi Square $(p \leq 0.05)$. No statistical significance relation to age and types of prostheses was observed. On RP, $47 \%$ was D and $53 \%$ DM, there was no statistically significant difference. It was possible to conclude that the rehabilitation of greater number of the teeth increases with age and is prevalent in all periods of adulthood, especially in women.

* $\quad$ Faculdade de Odontologia. Universidade de Ribeirão Preto. Ribeirão Preto, SP, Brasil.

** Centro de Investigación en Ciencias Odontológicas (CICO) Facultad de Odontología. Universidad de La Frontera. Temuco, Chile.

*** Departamento de Materiales Dentales y Prótesis. Universidad de São Paulo. Ribeirão Preto, SP, Brasil. 
Key words: Dental prostheses, prevalence, epidemiology.

Fecha de recepción: 15 de febrero de 2015.

Aceptado para publicación: 5 de mayo de 2015 .

de Andrade Lima Chaves C, Alves Gomes E, Faria T, Rocha Correa da Silva S, de Souza Filho CB, Borie E Corrêa Silva Sousa YT. Perfil de los pacientes rehabilitados por medio de prótesis atendidos en servicio público de salud en Ribeirão Preto, Brasil. Av. Odontoestomatol 2015; 31 (6): 347-353.

\section{INTRODUCCIÓN}

Actualmente, la caries es una enfermedad de alta prevalencia en los adultos de todos los países del mundo. Se ha observado que en países industrializados, más de la mitad de la población ha sido afectada, incrementando su predominio a medida que se aumenta la edad $(1,2)$. De acuerdo con la Organización Mundial de Salud (OMS), en las últimas décadas, algunos problemas como lesiones cariosas, enfermedad periodontal y edentulismo han aumentado en los países en desarrollo (3) y, por esta razón, se recomienda realizar estudios epidemiológicos en la población con edades entre 35-44 y 65-74 años, dado que los daños acumulados pueden ser causados en estos pacientes a los largo de la vida y, por consecuencia, influir en la calidad de vida y aumento del costo de la misma, tanto para los servicios de salud como para el paciente.

La Investigación Nacional en Salud Oral, realizada en Brasil el año 2010 (4), muestra que, en el rango etario entre los 35 y 44 años, el índice compuesto por la suma de dientes cariados, obturados y perdidos (COP-D) fue de 16,7 y la necesidad de algún tipo de prótesis existía en el 68,8\% de los casos, observando que una gran parte de la población $(41,3 \%)$ necesitaba de prótesis removible parcial o total, por lo menos, en uno de los arcos. Por otro lado, en el rango etario entre los 65 y 74 años, el índice COP-D fue de 27,5 , identificando que un $23,9 \%$ de los individuos necesitaba de una prótesis total en, por lo menos, uno de los maxilares y el $15,4 \%$ requería una prótesis total superior e inferior.

Urzua et al. (5), en el año 2012, publicaron un estudio donde evaluaron la prevalencia de caries en 1.553 adultos de origen chileno, entre las edades de 35 a 44 y 65 a 74 años. Los autores encontraron una alta prevalencia de caries y pérdida dental, observando índices de COP-D 15,06 y 21,57, respectivamente. Datos similares han sido reportados en estudios que analizaron la población del sur de Australia, entre los 45 y 54 años, y en los mayores de 60 años $(6,7)$, en la cual se observa que $50 \%$ de los adultos sufrieron de caries, siendo el factor más significante para pérdida dental y rehabilitaciones protésicas.

Se ha reportado que la mayoría de las lesiones cariosas son identificadas en los molares, presentando una alta frecuencia en su reconstrucción o ausencia de estas piezas dentarias posterior a los 60 años de edad $(7,8)$. Posterior al daño de un proceso carioso, el elemento dental puede ser rehabilitado mediante dos opciones: la restauración directa con resina compuesta o la confección de una prótesis dental. Sin embargo, esta última opción normalmente se indica cuando existe una gran destrucción coronaria $(9,10)$ y cuando se evalúa si el plan de tratamiento está de acuerdo a las condiciones socio-económicas del paciente $(10,11)$. Cuando la confección de una prótesis parcial fija pasa a ser la única opción de tratamiento, gran parte de los pacientes con baja renta económica opta por la remoción de la pieza dentaria, determinando una tendencia al edentulismo total en el rango etario de los 60 años o más (8).

Estudios epidemiológicos sobre el uso de prótesis dentales aún parecen ser escasos (11-13). Además, la correlación entre la edad y los tipos de prótesis realizadas en el servicio de atención pública brasileño aun no ha sido estudiado y esta información es de gran importancia, ya que la Política Nacional de 
Salud Oral en Brasil (14), incluyó en el año 2004 la rehabilitación protésica en el área de atención básica, con el objetivo de ampliar y calificar la atención especializada a la población de escasos recurso económicos y, principalmente, implantar Centros de Especialidades Odontológicas y Laboratorios Regionales de Prótesis Dentales.

De esta manera, con el fin de evaluar el perfil de los pacientes que buscan atención para rehabilitaciones dentales en el servicio público, el objetivo de este estudio fue analizar la distribución etaria y prevalencia de genero de los pacientes tratados en las clínicas de Prótesis Parcial Fija (PPF), Prótesis Parcial Removible (PPR) y Prótesis Total (PT), en un curso de Odontología durante un periodo de cinco años.

\section{MATERIAL Y MÉTODO}

Una evaluación retrospectiva y descriptiva fue realizada en 638 fichas clínicas de pacientes atendidos y tratados en las clínicas de Prótesis Parcial Fija (PPF), Prótesis Parcial Removible (PPR) y Prótesis Total (PT) de un curso de Odontología. Se investigaron todas las fichas de los pacientes que recibieron tratamiento durante un periodo de 5 años, específicamente, entre enero del 2008 hasta diciembre del 2013. Este estudio fue aprobado por el Comité de Ética de la Universidad de Ribeirão Preto (C.A.E.E. 30813014. 4.0000.5498).

Fueron incluidos en el estudio las fichas clínicas de pacientes rehabilitados mediante prótesis fijas, tanto unitarias como múltiples, en las cuales no importó si se utilizaron o no retenedores radiculares. Las PPR de los pacientes rehabilitados se clasificaron en dentomucosoportada (DM) o dentosoportadas (D), y aquellos pacientes que recibieron prótesis totales superior e inferior, se clasificaron según el arco donde fue indicado.

En cada ficha clínica se evaluaron los siguientes datos: género, edad y fecha de término del tratamiento, y para los pacientes con PPR, si su prótesis era DM o D. La PPR dentomucosoportada fue considerada cuando el paciente no posee los dientes posteriores, sea uni o bilateralmente, consiguiendo su retención en la mucosa y en el diente vecino al espacio desdentado, mientras que las PPRs dentosoportadas fueron consideradas cuando el espacio desdentado es intercalado entre dientes y es soportada por los dientes vecinos.

La tabulación y análisis de los datos fueron realizados por medio del software estadístico Graph Pad InStat v.3.6 (Graph Pad Software, Inc., San Diego, CA, USA). Para el análisis estadístico, los pacientes fueron divididos en 2 grupos en relación a su edad: A) $\geq 50$ años y B) $<50$ años. Las comparaciones entre los grupos fueron realizadas por el test exacto de Fisher y chi cuadrado, con nivel de significancia de $5 \%(p \leq 0,05)$.

\section{RESULTADOS}

De las 638 fichas clínicas de los pacientes atendidos en el Curso de Odontología, entre los años 20082013, 191 pacientes fueron atendidos en la clínica de PPF, 238 en la de PPR y 209 en la clínica de PT. Con relación a la edad, independiente del género, fue observado que para las rehabilitaciones con PPF, un $74 \%$ de los pacientes tenían menos de 50 años. En las clínicas de PPR y PT se identificó un predominio de personas mayores de 50 años (52\% y 92\%, respectivamente) (Figura 1). Con relación al género, independiente de la edad, se puede observar la prevalencia de mujeres atendidas para todos los tipos de prótesis (Figura 2).

La comparación entre edad y género de los pacientes atendidos en cada una de las clínicas de prótesis, realizadas por el Exacto de Fisher o chi cuadrado,

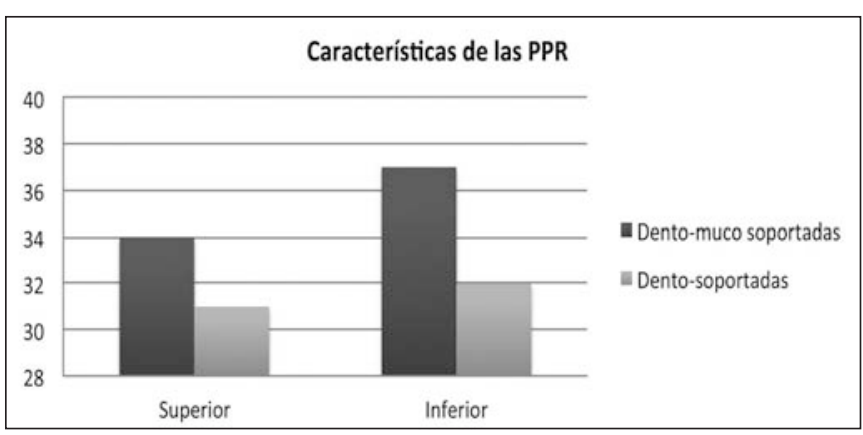

Fig. 1. Distribución del tipo de prótesis según el género. 


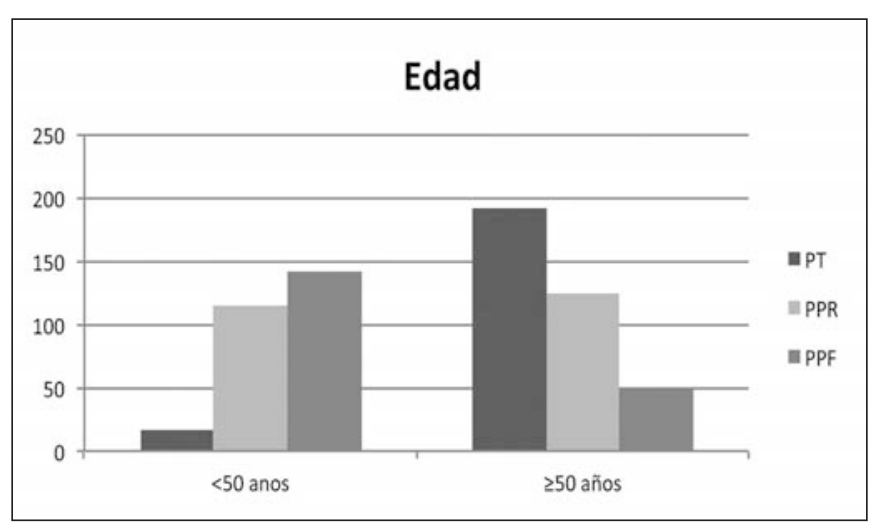

Fig. 2. Distribución del tipo de prótesis en la muestra según la edad.

dieron resultados estadísticamente significativos sólo para PPF $(p=0,02)$, sin identificar diferencias para PT $(p=1,01)$ ni para PPR $(p=0,145)$.

Además, se identificó mayor prevalencia de mujeres en la clínica de PT con edad $\geq 50$ años y en las clínicas de PPR y PPF hasta 50 años. Entre los hombres, se observó mayor prevalencia para pacientes usuarios de PT y PPR con edad $\geq 50$ años (Figura 3).

De las 238 fichas clínicas de pacientes atendidos en la clínica de PPR, solo una muestra de 134 individuos permitieron que las PPR fuesen clasificadas en D o DM, tanto para la arcada superior como la inferior. Se registró un mayor número de PPRs DM inferiores (37) y superiores (34), comparado a las D superiores (31) e inferiores (32). Sin embargo, no hubo diferencias significativas cuando se analizaron estos valores por el test de chi cuadrado $(p=0,97)$ (Figura 4).

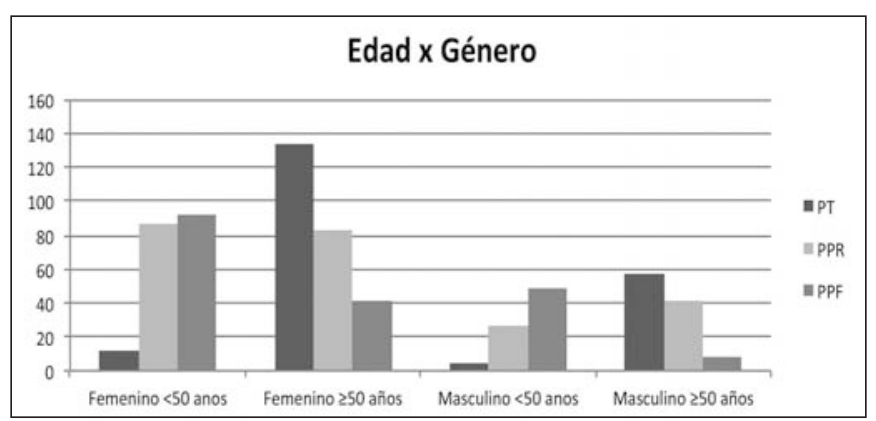

Fig. 3. Relación entre la edad y género según el tipo de prótesis.

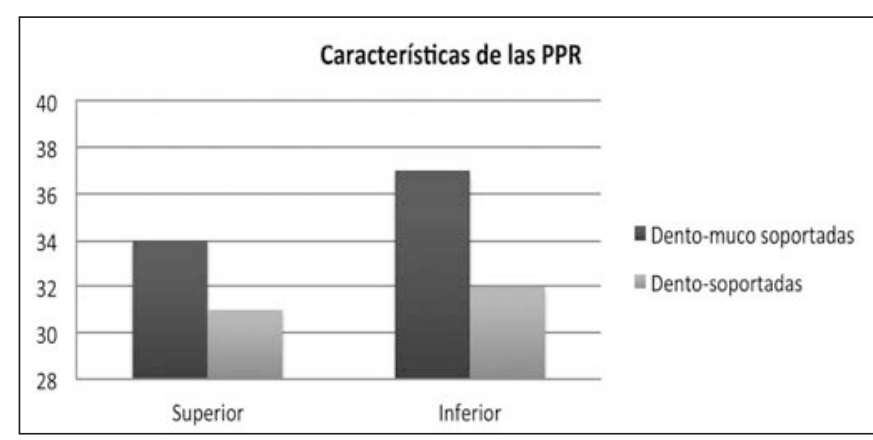

Fig. 4. Clasificación de las prótesis parciales removibles según el arco superior e inferior.

\section{DISCUSIÓN}

Según los últimos datos epidemiológicos nacionales que evaluaron la salud oral en la población de Brasil, se puede afirmar que la condición de salud bucal de los adultos brasileños ha mejorado en los últimos veinticinco años (15). A pesar de la disminución en la prevalencia de caries reportada en la última encuesta nacional de salud oral de la población adulta, las necesidades acumuladas aún son altas y este hecho puede estar asociado a la dificultad de acceso al tratamiento odontológico (16). Actualmente Brasil cuenta con la implantación de la política Nacional de Salud Oral, establecida en el año 2004 (14). A partir de ese momento, algunas estrategias fueron creadas para proporcionar a la población mayor cobertura en relación al tratamiento odontológico, como el inicio del programa "Brasil Sorridente", aumento de personal en los Equipos de Salud Oral, creación de la Estrategia de Salud de la Familia en el Sistema Único de Salud (SUS) y desarrollo de algunos Centros de Especialidades Odontológicas que ofrecen tratamientos de manera más especializada, tales como endodoncia y prótesis dentales (15).

En el presente estudio, durante un período de 5 años, 638 pacientes tuvieron acceso a las clínicas de prótesis del Curso de Odontología, recibiendo tratamiento especializado con prótesis parciales fijas y removibles. De ese total de individuos, 191 pacientes fueron atendidos en la clínica de PPF, 238 en la de PPR y 209 en la clínica de PT, observando una mayor prevalencia de pacientes de género femenino en todas las clínicas, identificando una edad inferior a 50 años en los pacientes rehabilitados con PPF y igual 
o superior a 50 años en aquellos rehabilitados con PPR y/o PT.

Una serie de estudios epidemiológicos que incluyen pacientes desdentados parciales o totales tanto en Brasil (16-19) como en otros países (11) han mostrado una clara prevalencia de pacientes del género femenino que solicitan tratamiento en las clínicas de prótesis, siendo bastante mayor que el género masculino, concordando de esta manera con los resultados encontrados en este estudio. El predominio individuos de género femenino en busca de tratamientos odontológicos rehabilitadores está directamente relacionado a las exigencias estéticas que se encuentran en la sociedad actual (20). Como las mujeres acuden más a las consultas de los cirujanos dentistas, en ellas se realizan más tratamientos de exodoncias y rehabilitaciones dentales en relación a los hombres (19). Además, por un factor económica y cultural, los hombres presentan más resistencia a frecuentar médicos y/o dentistas (21).

En un estudio sobre la prevalencia de restauraciones protésicas en adultos europeos, se observó que la frecuencia de uso de prótesis removible varió entre 13-29\%, identificando una reducción en las últimas décadas de usuarios de prótesis totales. Sin embargo, en países con menos desarrollo aún existe una mayor prevalencia de utilización de PTs (22).

Los resultados de este trabajo arrojaron que los pacientes rehabilitados con PPF poseen poca pérdida de piezas dentales en conjunto con una edad reducida ( $<50$ años), comparado con los pacientes portadores de PPR y PT. Tal resultado permite inferir que, esos adultos con edad inferior a los 50 años, en su gran mayoría son personas que se encuentran dentro del mercado laboral, con mayor exigencia estética y que forman parte de un grupo con "alto capital social" (formal o informal). En este sentido, las personas que pertenecen al grupo de alto capital social pueden tener mayores posibilidades de tratamiento de la enfermedad de caries ya instalada, evitando la pérdida de sus piezas dentales y/o presentando pocos dientes perdidos, relacionados al mayor soporte social existente en esos grupos (15). Así, la decisión por el tipo de prótesis a ser utilizada por el paciente termina siendo directamente dependiente del número de dientes ausentes, observando que cuando son pocos los dientes perdidos, los pacientes optan por PPF, y por el contrario, cuando existe una menor cantidad de piezas dentales hay un claro predominio de elección de prótesis removible (22).

Otro punto a destacar de este estudio fue factor edad, específicamente en pacientes con edad igual o mayor a 50 años, en los cuales se identificaron una mayor cantidad de dientes ausentes. En esta esfera, los individuo comenzaron requiriendo PPR y, posteriormente, terminaron utilizando PT. Esta "involución" en relación a los tipos de prótesis muchas veces está directamente asociada a factores educacionales, motivacionales e higiénicos, tanto del clínico como del paciente (10).

Otros factores que también pueden estar asociados a una mayor cantidad de dientes ausentes en pacientes con edad igual o mayor a los 50 años guarda relación con el "bajo capital social", el cual se trata de individuos inseguros y que no participan de ningún grupo social, sea formal o informal (15). Es importante destacar que los pacientes analizados fueron derivados de la Secretaría Municipal de Salud, obteniéndose predominantemente un grupo con bajo nivel de escolaridad y con condiciones socio-económicas poco favorables.

Individuos de baja renta que viven en barrios más pobres o en ciudades con menor índice de desarrollo humano, pierden una mayor cantidad de piezas dentales comparados con aquellos que viven en barrios y/o ciudades más desarrolladas, lo cual es explicado, principalmente, por la renta per cápita $(16,23,24)$. Algunas condiciones sociodemográficas negativas también están relacionadas a una mayor frecuencia de pérdida dental ya que en muchos casos no ofrecen otro tipo de terapias para tratar la enfermedad (8).

Con la pérdida de un mayor número de dientes, los paciente económicamente menos favorecidos y que no pueden costear tratamientos rehabilitadores sobre prótesis implantosoportadas, optan por rehabilitaciones con PPR, por el hecho de presentar un menor costo y ser menos invasivo si se compara con las PPFs (25). En este estudio, no existieron diferencias significativas para los tipos de prótesis dentosoportada o dentomucosoportada, sugiriendo que no hay una 
característica específica en el padrón de pérdida de los elementos dentales en la población evaluada.

Con el uso de PPR, pueden suceder algunas alteraciones no deseadas en la cavidad oral, tal como cambios en la microbiología y aumento en la retención de placa bacteriana en los dientes de soporte, lo que puede aumentar el riesgo de gingivitis, periodontitis o caries (25), consiguiendo, posiblemente, la pérdida de la pieza dental y alteración de la distribución de los espacios desdentados a medida que pasa el tiempo (19). Debido a estos factores de alteración de la cavidad oral como consecuencia del uso de PPR, es que se puede llegar al edentulismo total.

En Brasil, el tratamiento protésico ofrecido por el servicio público, es ejecutado por cursos de Odontología, a través de convenios con los servicios del Sistema de Salud, y esto es una de las limitaciones de este estudio, ya que el análisis fue realizado en un solo servicio. Sin embargo, creemos que los resultados son importantes porque muestran las condiciones orales y protésicas de un grupo poblacional que se ha tornado importante durante los últimos años. Por lo anterior, controles de mantención de los tratamientos protésicos deben ser ofrecidos por los servicios de salud para que no ocurra, con la evolución de la edad, un aumento en el número de dientes extraídos. Así, pacientes rehabilitados con PPF no tendrán que ser necesariamente rehabilitados posteriormente con PPR o PT.

\section{CONCLUSIÓN}

De acuerdo con los resultados obtenidos en el presente estudio se puede concluir que la necesidad de rehabilitaciones de las piezas dentales aumenta en relación con la edad, siendo prevalente en todos los periodos de la vida adulta, principalmente en el género femenino, pudiendo estar relacionado este hecho a condiciones culturales y socio-económicas de este grupo poblacional.

\section{BIBLIOGRAFÍA}

1. Petersen P, Razanamihaja N, Poulsen VJ. Surveillance of Oral Health among Children and
Adults in Madagascar, WHO, Geneva, Switzerland, 2004.

2. Griffin SO, Griffin OM, Swann JL, Zlobin N. New coronal caries in older adults: implications for prevention. J Dent Res 2005;84(8):715-20.

3. Burt BA. Concepts of risk in dental public health. Community Dent Oral Epidemiol 2005;33(4): 240-7.

4. Brasil. Ministério da Saúde. SB Brasil 2010. Pesquisa Nacional de Saúde Bucal. Resultados principais. Brasília, 2011, 92p.

5. Urzua I, Mendoza C, Arteaga O, Rodríguez G, Cabello R, Faleiros $\mathrm{S}$, et al. Dental caries prevalence and tooth loss in chilean adult population: first national dental examination survey. Int J Dent 2012;2012:810170.

6. Brennan DS, Spencer AJ, Roberts-Thomson KF. Caries experience among 45-54 year olds in Adelaide, South Australia. Aust Dent J 2007;52(2):122-7.

7. Slade GD, Spencer AJ. Distribution of coronal and root caries experience among persons aged 60+ in South Australia. Aust Dent J 1997;42(3):178-84.

8. Gaio EJ, Haas AN, Carrard VC, Oppermann RV, Albandar J, Susin C. Oral health status in elders from South Brazil: a population-based study. Gerodontology 2012;29(3):214-23.

9. Janus CE, Unger JW, Best Al M. Survival Analysis of Complete Veneer Crowns vs. Multisurface Restorations: A Dental School Patient Population. J Dent Educ 2006;70(10):1098-104.

10. De Backer H, Van Maele G, Decock V, Van den Berghe L. Long-term survival of complete crowns, fixed dental prostheses, and cantilever fixed dental prostheses with posts and cores on root canal-treated teeth. Int J Prosthodont 2007;20(3): 229-34.

11. Näpänkangas R, Haikola B, Oikarinen K, Söderholm AL, Remes-Lyly T, Sipilä K. Prevalence of single crowns and fixed partial dentures in 
elderly citizens in the southern and northern parts of Finland. J Oral Rehabil 2011;38(5):328-32.

12. Ranta K, Tuominen R, Paunio I. Rehabilitation with fixed prostheses among Finnish adults. Community Dent Health1986;4(2):137-42.

13. Pine CM, Pitts NB, Steele JG, Nunn JN, Treasure E. Dental restorations in adults in the UK in 1998 and implication for the future. Br Dent $\mathrm{J} 2001 ; 190$ (1):4-8.

14. Brasil. Ministério da Saúde. Diretrizes da Política Nacional de Saúde Bucal. Brasília, 2004, 16p.

15. Borges CM, Campos ACV, Vargas AMD, Ferreira EF. Perfil das perdas dentárias em adultos segundo o capital social, características demográficas e socioeconômicas. Ciênc saúde coletiva [online] 2014;19(6):1849-58.

16. Rihs LB, Da Silva DD, Sousa MLR. Dental caries in an elderly population in brazil. J Appl Oral Sci 2009;17(1):8-12.

17. Carneiro RMV, Silva DD, Sousa MLR, Wada RS. Oral health of institutionalized elderly in the Eastern Zone of São Paulo, Brazil, 1999. Cad Saude Publica 2005;21(6):1709-16.

18. Giordani JMA, de Slavutzky SMB, Koltermann AP, Pattussi MP. Inequalities in prosthetic rehabilitation among elderly people: the importance of context. Community Dent Oral Epidemiol 2011;39(3): 230-8.

19. Pellizzer EP, Almeida DAF, Falcon-Antenucci RM, Sánchez DM, Zuim PRJ, Verr FR. Prevalence of removable partial dentures users treated at the Aracatuba Dental School - UNESP. Gerodontology 2012;29(2):140-4.
20. Carvalho G, Rosemburg CP, Buralli KO. Avaliação de ações e serviços de saúde. Mundo Saúde 2000; 24(1):72-88.

21. Martins MATS, Mendonça SMS, Vargas AMD. Tratamento odontológico na rede pública de Belo Horizonte: percepção de cirurgiões-dentistas e alunos. Rev ABO Nac 1999;6(1):362-6.

22. Zitzmann NU, Hagmann E, Weiger R. What is the prevalence of various types of prosthetic dental restorations in Europe? Clin Oral Impl Res 2007; 18:20-33.

23. Turrell G, Sanders AE, Slade GD, Spencer AJ, Marcenes W. The independent contribution of neighborhood disadvantage and individual-level socioeconomic position to self-reported oral health: a multilevel analysis. Community Dent Oral Epidemiol 2007;35(3):195-206.

24. Sanders AE, Turrell G, Slade GD. Affluent neighborhoods reduce excess risk for tooth loss among the poor. J Dent Res 2008;87(10):96973.

25. Budtz-Jörgensen E. Restoration of the partially edentulous mouth -a comparison of overdentures, removable partial dentures, fixed partial dentures and implant treatment. J Dent 1996;24(4):23744.

\section{CORRESPONDENCIA}

Yara Teresinha Corrêa Silva Sousa

Facultad de Odontología

Universidad de Ribeirão Preto

14024-070, Ribeirão Preto, SP, Brasil

E-mail:ysousa@unaerp.br 